\title{
Relato de Experiência Docente:A Primeira Disciplina no Brasil sobre a Prática da Psicologia Baseada em Evidências Ministrada no Instituto de Psicologia da Universidade de São Paulo
}

\author{
Tamara Melnik ${ }^{1,2, *}$ (D), Sonia Beatriz Meyer $^{3}$, \& Maria Imaculada Cardoso Sampaio ${ }^{4}$ \\ ${ }^{1}$ Programa de Saúde Baseada em Evidências, Universidade Federal de São Paulo, São Paulo, SP, Brasil \\ ${ }^{2}$ Centro Cochrane do Brasil, São Paulo, SP, Brasil \\ ${ }^{3}$ Instituto de Psicologia da Universidade de São Paulo, São Paulo, SP, Brasil \\ ${ }^{4}$ Universidade Santo Amaro, São Paulo, SP, Brasil
}

\begin{abstract}
RESUMO - Este artigo relata a experiência de ensino da primeira disciplina no Brasil sobre a Prática da Psicologia Baseada em Evidências (PPBE), oferecida pelo Instituto de Psicologia da Universidade de São Paulo. O curso instruiu os alunos sobre os conceitos da PPBE, fontes de evidências primárias e secundárias, adequação da pergunta de pesquisa ao delineamento, delineamento experimental de caso único, estudos observacionais, ensaios clínicos randomizados e sobre a execução de revisões sistemáticas, com ou sem metanálise. É importante destacar que o desenvolvimento da disciplina oportunizou a reflexão sobre a importância da PPBE no âmbito clínico, acadêmico, institucional e na gestão de políticas públicas na saúde mental. Recomenda-se a inserção da disciplina na grade curricular dos cursos de graduação e pósgraduação na área da Psicologia
\end{abstract}

PALAVRAS-CHAVE: psicologia baseada em evidências, revisão sistemática, formação do psicólogo

\section{Teaching Experience Report:The First Discipline in Brazil on the Evidence-Based Practice in Psychology Offered by the Institute of Psychology of the University of São Paulo}

\begin{abstract}
This article reports the experience of teaching the first course in Brazil on the Evidence-Based Practice in Psychology (EBPP), offered by the Institute of Psychology of the University of São Paulo. The course instructed students on the concepts of EBPP, primary and secondary sources of evidence, adequacy of the research question to the experimental design, single-subject design, observational studies, randomized controlled trials and implementation of systematic reviews with or without meta-analysis. It is noteworthy that the development of the discipline allowed reflections on the importance of EBPP in clinical, academic, institutional and public policy management on mental health. It is recommended the inclusion of EBPP course in the curriculum of undergraduate and graduate students in Psychology.
\end{abstract}

KEYWORDS: evidence-based psychology, systematic reviews, psychologist education

A disciplina Introdução à Prática da Psicologia Baseada em Evidências (PPBE) foi concebida pela necessidade de permitir o acesso dos alunos de pós-graduação do Instituto de Psicologia da Universidade de São Paulo aos princípios da PPBE. A demanda por PPBE faz-se primordial na atualidade, uma vez que é urgente que as práticas envolvidas na prevenção, cuidado e promoção à saúde sejam baseadas nos resultados das melhores evidências científicas. O profissional da saúde não pode fundamentar a sua prática clínica apenas na sua formação universitária, experiência profissional e intuição, sem recorrer às evidências científicas para apoiar a sua tomada de decisão (Melnik, Souza, \& Carvalho, 2014).

É indiscutível os desafios enfrentados pelos sistemas de saúde em diferentes países, principalmente no Brasil,

*E-mail: tamelnm@gmail.com

- Submetido: 25/05/2016; Revisado: 04/08/2017; Aceito: 16/02/2018. 
frente às decisões sobre incorporação de alternativas preventivas e terapêuticas na saúde mental. Escolhas difíceis são enfrentadas por psicólogos, psiquiatras, acadêmicos e gestores em todos os níveis do sistema de saúde mental. O arsenal de intervenções na atenção à saúde mental é vasto, sendo continuamente ampliado com diversas alternativas psicoterápicas, novos medicamentos e modalidades de tratamentos presencias e, mais recentemente, on-line. Essa realidade faz com que, a cada ano, torne-se mais difícil para o profissional e para o sistema fornecer ao usuário intervenções com provas científicas de efetividade, eficácia e segurança. A PPBE é ainda mais necessária em países em desenvolvimento, como o Brasil, em razão da escassez de recursos financeiros. A utilização de intervenções psicossociais sem evidências de benefício, ou comprovadamente ineficazes, é absolutamente injustificável.

Frente a esse cenário, em 2006, a American Psychological Association (APA) reuniu um grupo de cientistas e psicólogos clínicos, não privilegiando nenhuma abordagem teórica, e criou a Força Tarefa em Práticas Baseadas em Evidências (Task Force on Evidence Based Practice - TFEBP). O relatório desse grupo define a PPBE como a integração da melhor evidência disponível na pesquisa à experiência clínica do psicólogo, contextualizando as características individuais e culturais dos clientes/pacientes, bem como as suas preferências. A proposta é a aplicação dos princípios da PPBE a diversos campos de atuação: clínica, diagnóstica, supervisão, hospitalar, institucional e acadêmica (Levant, 2005).

Cabe ressaltar que a evidência científica não exclui, de todo, as incertezas. Intervenções psicoterápicas são passíveis de sofrer influências de diversas variáveis, como características do terapeuta (tempo de formação, especialização, supervisão) e características do paciente (comorbidades orgânicas e psíquicas, nível socioeconômico, motivação), nem sempre consideradas a priori nos estudos. Nesse sentido, ainda que as tomadas de decisão de programas de intervenção e políticas de saúde mental venham a ser baseadas em evidências, essas decisões devem ser alvo permanente de processos de monitoramento e avaliação sistemáticos, visando a correção e o aprimoramento deles, de acordo com seus resultados (Melnik \& Atallah, 2011). Portanto, a PPBE demanda do psicólogo constante atualização e conhecimento dos princípios metodológicos envolvidos na pesquisa (Chorpita \& Daleiden, 2004).

A prática da saúde baseada em evidências, em qualquer campo de atuação das áreas da Psicologia, Medicina, Enfermagem e Odontologia, contempla conceitos da Epidemiologia Clínica, ou seja, da aplicação de princípios e métodos epidemiológicos e técnicas estatísticas para o estudo de questões clínicas e melhoria dos cuidados clínicos.

Portanto, a expressão Psicologia Baseada em Evidências, assim como Psicoterapia ou Terapia Baseada em Evidências, não é um "rótulo" oportuno, e sim, um construto que envolve a compreensão da hierarquia das evidências na Psicologia, da avaliação crítica das evidências, dos métodos aplicados no desenvolvimento de revisões sistemáticas, das metanálises, dos ensaios clínicos randomizados, e demais delineamentos experimentais bem conduzidos, entre outros domínios. Diante da complexidade da PPBE, é necessário o treinamento de graduandos e pós-graduandos para que os princípios que a fundamentam possam ser uma possibilidade de avanço do conhecimento científico e considerados em todos os campos da Psicologia.

Desde a década de 90 do século XX, existe o consenso de que as melhores fontes de evidências sobre tratamento e prevenção em saúde mental são as revisões sistemáticas (Guyatt et al., 1992). Tais estudos vêm sendo utilizados na base de elaboração de diretrizes de avaliações tecnológicas em saúde. Esse tipo de estudo também tem se mostrado extremamente útil no direcionamento de recursos para pesquisas e na proposição de programas de intervenção em saúde pública (Melnik \& Atallah, 2011).

Recentemente, em 2015, a APA publicou a atualização dos critérios para a classificação dos tratamentos psicológicos com sustentação científica. Essa lista está disponibilizada no site da Divisão 12 de Psicologia Clínica da APA. Uma das principais modificações dessa atualização foi a inclusão das revisões sistemáticas, seguidas de metanálises, como nível I na tomada de decisão na Psicologia, além da utilização do GRADE (Grading of Recommendations, Assessment, Development and Evaluation), um sistema desenvolvido por um grupo colaborativo de pesquisadores que visa a criação de um sistema universal, transparente e sensível para graduar a qualidade das evidências e a força das recomendações. No GRADE, a qualidade da evidência é classificada em quatro níveis: alto, moderado, baixo, muito baixo. A força da recomendação expressa a ênfase para que seja adotada ou rejeitada uma determinada intervenção psicoterápica, considerando potenciais vantagens - efeitos benéficos na melhoria dos sintomas psíquicos, qualidade de vida e redução dos custos - e desvantagens - riscos de efeitos adversos, a carga psicológica para o paciente e seus familiares e os custos para a sociedade. $O$ balanço na relação entre vantagens e desvantagens determina a força da recomendação.

Portanto, para que os psicólogos utilizem o conhecimento na sua prática profissional e tenham amplo domínio de metodologia científica para uma interface produtiva entre ciência e prática, é necessário que se inclua, na formação do aluno, competências para lidar com problemas de amostragem, observação, registro, interpretação de dados, análises estatísticas, níveis de evidências, revisões sistemáticas, metanálises e avaliação crítica de artigos científicos (Witter, 2007).

Considerando os aspectos destacados, o objetivo deste relato de experiência é demonstrar como a disciplina Introdução à PPBE foi estruturada e oferecida aos alunos do curso de pós-graduação em Psicologia Clínica do Instituto de Psicologia da Universidade de São Paulo (IPUSP). O compartilhamento do conteúdo e da forma como o curso foi organizado foram detalhadas para que outras instituições possam reproduzir a experiência com seus estudantes. 


\section{OBJETIVOS DA DISCIPLINA}

\section{Objetivo Geral}

A disciplina tinha como objetivo geral introduzir os princípios da PPBE no diagnóstico, tratamento e prevenção de recaídas nos diversos campos de atuação profissional do psicólogo: clínico, hospitalar, institucional e acadêmico.

\section{Objetivos Específicos}

Para capacitar o profissional, a disciplina compreendeu os seguintes objetivos específicos: (1) realizar buscas sistematizadas das melhores evidências da literatura científica; (2) avaliar criticamente a literatura da Psicologia; (3) aprimorar conhecimentos de epidemiologia e metodologia de pesquisa; (4) elaborar e executar projetos de pesquisa nos preceitos da PBE; e (5) praticar a PBE.

\section{MÉTODO DA DISCIPLINA}

A disciplina foi oferecida no segundo semestre do ano de 2013 aos alunos de pós-graduação do programa de PósGraduação do IPUSP, compreendeu 14 aulas, perfazendo um total de 70 horas. Participaram da disciplina nove alunos regularmente matriculados. O conteúdo, as referências e as tarefas desenvolvidas na disciplina estão apresentados na Tabela 1 .

Além das aulas expositivas, exercícios e leituras complementares, outro elemento essencial na formação do psicólogo para o uso da informação na tomada de decisão é a capacitação para o uso das fontes de informação. A área de Psicologia, devido à interdisciplinaridade de seu objeto de estudo e pesquisa, conta com diversas bases de dados e possibilidades de busca de informação. O desenvolvimento de estratégias de busca sensibilizadas, com o uso de palavras-chave adequadas e que esgotem as possibilidades de mapeamento do tema são elementos essenciais na PPBE. Dessa maneira, a parte prática de capacitação dos alunos nesses quesitos foi de grande valia para o sucesso da disciplina.

Ao final da disciplina foi realizada a avaliação da relevância do tema na graduação e pós-graduação, do conteúdo ministrado e técnica de ensino, a qual permitiu aos alunos uma participação anônima. Os resultados gerados foram compilados de acordo com as categorias relevância, conteúdo e técnica de ensino e embasaram a proposta de uma disciplina obrigatória na pós-graduação.

\section{DISCUSSÃO E CONSIDERAÇÕES FINAIS}

A metodologia da disciplina, com aulas teóricas, leituras, tarefas e parte prática na elaboração de estratégias de busca e aplicação às bases de dados foi considerada adequada por todos os participantes. A sugestão para que a disciplina fosse incorporada à grade curricular da formação dos alunos de pós-graduação nos demais programas do IPUSP foi fornecida por diversos alunos, tanto na avaliação formal, quanto informalmente, durante o desenvolvimento da disciplina.

Conforme mencionamos anteriormente, a demanda pela prática na saúde baseada em evidências é crescente nos diferentes campos de atuação profissional dessa área: pesquisa, prevenção e assistência. Nesse sentido, é consensual que a Psicologia pode se beneficiar da incorporação dessa ferramenta no seu trabalho. Os clínicos podem utilizar a revisão sistemática para se manterem bem informados, os pesquisadores podem utilizá-la para identificar, justificar e formular mais hipóteses de pesquisas e os administradores da saúde podem usá-la para a formulação de diretrizes e legislação necessárias para diagnósticos e estratégias de tratamento e prevenção da saúde (Melnik \& Atallah, 2011).
Atualmente, o acesso à informação tem sido ampliado por meio da disponibilização de um grande volume de conhecimento científico na internet. Para que o psicólogo possa utilizar de forma adequada a produção cientifica é necessária a apropriação de conhecimentos de epidemiologia, metodologia, revisões sistemáticas, estatística e avaliação crítica da literatura. Na disciplina Introdução PPBE, os alunos tiveram a oportunidade de percorrer alguns conceitos fundamentais para a PBE: aspectos conceituais e históricos da PBE, estruturação da pergunta de pesquisa, tipos de delineamentos experimentais, avaliação crítica da literatura, revisões sistemáticas, diretrizes e elaboração de um projeto de pesquisa nos preceitos da prática na saúde baseada em evidências.

Conforme apontado anteriormente, as vantagens da PBE vêm desde o planejamento de pesquisas em temas prioritários na Psicologia, para se evitar desperdícios no fomento à pesquisa, até o auxílio na tomada de decisão na clínica e na gestão de saúde pública. Os alunos destacaram a importância da aquisição desse conhecimento, bem como a necessidade da incorporação da disciplina PPBE em nível de graduação e pós-graduação. 
Tabela 1

Grade e referências da disciplina Introdução à Psicologia Baseada em Evidências

\begin{tabular}{ll}
\hline Aulas & \multicolumn{1}{c}{ Leituras (ver Referências para detalhes) e Tarefas } \\
\hline $\begin{array}{l}\text { 1. Psicologia Baseada em } \\
\text { Evidências: aspectos conceituais } \\
\text { e históricos }\end{array}$ & $\begin{array}{l}\text { Melnik, T., \& Atallah A. N. (2011). Psicologia baseada em evidências: Provas científicas da efetividade da } \\
\text { psicoterapia. São Paulo: Editora Santos. }\end{array}$
\end{tabular}

2. Formulação da pergunta de pesquisa PICOT

3. Fontes primárias e fontes secundárias de boas evidências científicas na Psicologia

4. Relato de caso e delineamento experimental de caso único

5. Estudos observacionais na Psicologia: coorte, caso controle, série de casos

6. Ensaios Clínicos

Randomizados na Psicologia: definição, planejamento, execução e avaliação crítica

7. Revisões sistemáticas e metanálise na Psicologia

8. Revisões sistemáticas e metanálises na Psicologia (I)

9. Revisões sistemáticas e metanálises na Psicologia (II)

10. Guidelines na Saúde Mental

11. Avaliação Crítica da Literatura Psicológica

12. Apresentação dos projetos
Stewart, R. E., \& Chambless, D. L. (2007). Does psychotherapy research inform treatment decisions in private practice? Journal of Clinical Psycholology, 63(3), 267-81.

Chambless, D. L. (1996). In defense of dissemination of empirically supported psychological interventions. Clinical Psychology Science and Practice, 3(3), 230-235. doi:10.1111/j.1468-2850.1996.tb00074.x

Sampaio, M. I. C., \& Sabadini, A. A. Z. P. (2009). Indexação e fator de impacto. In A. A. Z. P. Sabadini, M. I. C Sampaio, \& S. H. Koller (Orgs.), Publicar em psicologia: Um enfoque para a revista cientifica (pp. 103-116). São Paulo: Associação Brasileira de Editores Científicos de Psicologia, IPUSP. Recuperado de http://www. ip.usp.br/biblioteca/pubcursos/publicar psicologia 1 edicao 2009 WEB COR 13\%20jul\%202009.pdf Cochrane Report Reviews. Mostly of these are from Medicine area, but there are some about mental disorders, largely severe mental illness. Recuperado de http://www3.interscience.wiley.com/cgi-bin/mrwhome/1065687 53/HOME

Tarefa: Dentro do tema de investigação, elaborar uma estratégia de busca e citar as bases que serão consultadas.

Oshiro, C. K. B., Kanter, J. W., \& Meyer, S. B. (2012). A single-case experimental demonstration of functional analytic psychotherapy with two clients with severe interpersonal problems. International Journal of Behavioral Consultation and Therapy, 7(2-3), 111-116.

Sampaio, A. A. S., de Azevedo F. H. B., Cardoso, L. R. D., de Lima, C., Pereira, M. B. R., \& Andery, M. A. P. A. (2008). Uma introdução aos delineamentos experimentais de sujeito único. Interação em Psicologia, 12(1), 151164. Recuperado de http://ojs.c3sl.ufpr.br/ojs index.php/psicologia/article/viewFile/9537/9218

Tarefa: Dentro do tema de investigação, elaborar pergunta de pesquisa que seria respondida por relato de caso e por delineamento experimental de caso único. Apresentar um esboço de método.

Mann, C. J. (2003). Observational research methods. Research design II: cohort, cross sectional and case-control studies. Emergency Medicine Journal, 20, 54-60. doi:10.1136/emj.20.1.54

Tarefa: Dentro do tema de investigação, elaborar pergunta de pesquisa que seria respondida por um estudo de coorte e por um estudo caso controle. Apresentar um esboço de método.

Kendall, J. M. (2003). Designing a research project: Randomised controlled trials and their principles. Emergency Medicine Journal, 20(2), 164-168. doi: 10.1136/emj.20.2.164

Tarefa: Dentro do tema de investigação, elaborar pergunta de pesquisa que seria respondida por um ensaio clínico randomizado. Apresentar um esboço de método.

Stewart, R. E., \& Chambless, D. L. (2010). Interesting practitioners in training in empirically supported treatments: Research reviews versus case studies. Journal of Clinical Psychology, 66(1), 73-95. doi:10.1002/ jclp. 20630

Tarefa: Elaborar uma ficha de extração de dados.

Greenhalgh, T. (1997). How to read a paper: Papers that summarise other papers (systematic reviews and metaanalyses). British Medical Journal, 315(7109), 672-675.

Higgins, J. P. T., \& Green, S. (2006.) Cochrane handbook for systematic reviews of interventions (Version 5.1.0). Chichester, UK: John Wiley \& Sons. Recuperado de http://www.cochrane.org/ sites/default/files/uploads/ Handbook4.2.6Sep2006.pdf

Tarefa: Avaliação do risco de viés de um estudo primário incluído em uma revisão sistemática.

Melnik, T., Hawton, K., \& McGuire, H. (2012). Interventions for vaginismus. Cochrane Database Systematic Review, 12. doi: 10.1002/14651858.CD001760.pub2.

NICE. (2011). Common mental health disorders: Identification and pathways to care. NICE Clinical Guideline, 123. Recuperado de www.nice.org.uk/CG123 [NICE guideline].

Tarefa: Sumarizar os dados de um guideline relacionado com o seu tema de pesquisa.

Altman, D. G., Schulz, K. F., Moher, D., Egger, M., Davidoff, F., Elbourne, Elbourne, D., ... \&Lang, T. (2001). The revised CONSORT statement for reporting randomized trials: Explanation and elaboration. Annals of Internal Medicine, 134(8), 663-694.

Shea, B. J., Grimshaw, J. M., Wells, G. A., Boers, M., Andersson, N., Hamel, C., ... Bouter, L. M. (2007). Development of AMSTAR: A measurement tool to assess the methodological quality of systematic reviews. BMC Medical Research Methodology, 7(1), 10. doi:10.1186/1471-2288-7-10

Tarefa: Realizar uma avaliação crítica de um ensaio clinico randomizado e de uma revisão sistemática.

Elaboração de um projeto de pesquisa nos preceitos da Prática na Saúde Baseada em Evidências. 
Conforme os alunos ressaltaram, "a Psicologia tem de sair do achismo" e buscar respeitabilidade frente às outras especialidades na área da Saúde por meio de uma produção científica consistente e uma postura clínica baseada na experiência, preferências do paciente (cliente) e evidências científicas.

O paradigma da PPBE tende a se difundir. Avanços na divulgação do conhecimento estão facilitando as mudanças necessárias para sua adoção (Melnik \& Atallah, 2011). Em países que começam a mostrar sinais da PPBE, por exemplo, Canadá, Estados Unidos e Inglaterra, sua aceitação de forma mais ampla se deve à vontade e determinação política de líderes de revistas, faculdades de psicologia, sociedades científicas e serviços de saúde (Chorpita \& Daleiden, 2004; Roth \& Fonagy, 2005).

O Brasil apresentou poucas iniciativas em PBE até o momento, sendo notória a necessidade de operacionalização e divulgação. Em 2011, foi publicado o primeiro livro nacional sobre PBE, Psicologia baseada em evidências: provas cientificas da efetividade da psicoterapia, organizado por de Melnik e Atallah (2011). Escrito a várias mãos e, indubitavelmente, por alguns dos maiores especialistas na área da saúde mental do Brasil, o livro abrange, dentre outros temas, o tratamento psicoterápico (isolado e/ou associado a psicofármacos) em diversos transtornos mentais.

A disseminação da PPBE propõe uma forma de estabelecer um diálogo e otimizar a comunicação entre gestores, autoridades de saúde pública, tomadores de decisão, agências de fomento à pesquisa, profissionais de saúde, pesquisadores, pós-graduandos e universidades que estejam buscando conhecimento que poderia servir de base para suas pesquisas.

Não sendo a Psicologia mágica, nem divina, seu desenvolvimento deve se apoiar no saber científico e na arte de aplicá-lo para o bem dos pacientes (Roth \& Fonagy, 2005). A aceitação - por profissionais envolvidos na saúde mental - das incertezas do saber científico e, desse modo, da falibilidade, é o ponto de partida na construção da prática psicológica crítica que, por conseguinte, se renovará continuamente em prol da melhoria da qualidade de vida das pessoas.

\section{REFERÊNCIAS}

American Psychological Association - APA. (2006). Evidencebased practice in psychology: APA presidential task force on evidence-based practice. American Psychologist, 61, 271-285.

Chorpita, B. F., \& Daleiden, E. L. (2004). Designs for instruction, designs for change: Distributing knowledge of evidencebased practice. Clinical Psychology: Science and Practice, 11, 332-335.

Guyatt, G., Cairns, J., Churchill, D., Cook, D., Haynes, B., Hirsh, J., ... Sackett, D. (1992). Evidence-based medicine: A new approach to teaching the practice of medicine. Jama, 268(17), 2420-2425.

Levant, R. F. (2005). Report of the 2005 task force on evidencebased practice. Recuperado de http://www.apa.org/practice/ resources/evidence/evidence-based-report.pdf

Melnik, T., \& Atallah, A. (2011). Psicologia baseada em evidências: Articulação entre a pesquisa e a prática clínica. In T. Melnik
\& A. N. Atallah (Orgs.), Psicologia baseada em evidências: Provas cientificas da efetividade da psicoterapia (pp. 3-13). São Paulo: Santos.

Melnik, T., Souza, W. F., \& Carvalho, M.R. (2014). A importância da prática da psicologia baseada em evidências: Aspectos conceituais, níveis de evidência, mitos e resistências. Revista Costarricense de Psicología, 33(2), 79-92.

Roth, A., \& Fonagy, P. (2005). What works for whom? A critical review of psychotherapy research ( $2^{\mathrm{a}}$ Ed.). New York: Guiford.

Witter, G. P. (2007). Psicoterapia: Pesquisa e prática. [Resenha do livro Evidence-based psychoterapy: Where practice and research meet, de C. D. Goodheart, A. E. Kazdin, \& R. J. Sternberg (Eds.)]. Psicologia: Reflexão e Crítica, 20(2), 523-524. doi: http://dx.doi.org/10.1590/S010279722007000300021 